\title{
Composición de lianas y bejucos en el Chocó Biogeográfico Colombiano
}

\author{
Composition of lianas and vines in the Colombian Chocó
}

\author{
Deivis Abadia Bonilla ${ }^{1,2}$, Álvaro de Jesus Lema Tapia ${ }^{2}$ \& Luz Yorleyda Palacios-Tello ${ }^{1}$
}

\begin{abstract}
Resumen
Con el objetivo de determinar los patrones de distribución de lianas y bejucos en el Chocó biogeográfico colombiano, se construyó una base en datos de presencia de las especies de lianas y bejucos reportadas a diciembre de 2012, a partir de bases de datos digitales, registros en literatura científica e inspección directa de especímenes del herbario teniendo en cuenta los tipos de ecosistemas (ambientes), a partir de la cual, se determinó la composición y riqueza para cada tipo de ambiente. Para evaluar la afinidad en términos de composición de familias entre tipos de ambientes, se elaboró un análisis de agrupamiento jerárquico, utilizando el coeficiente de similaridad de Jaccard. Se registraron 41 familias, distribuidas en 198 géneros y 700 especies de lianas y bejucos para el Chocó Biogeográfico. Las familias mejor representadas en cuanto a número de especies y géneros fueron Bignoniaceae (75/34), Fabaceae (74/19), Sapindaceae (59/4) y Rubiaceae (40/14). Existe una similaridad del 72\% en la composición de lianas y bejucos respecto a los tipos de ambiente del Chocó Biogeográfico colombiano.

Palabras clave: Presencia-ausencia, similaridad, Bignoniaceae, trepadoras, diversidad.

Abstract

In order to determine the distribution patterns of lianas and vines in the Colombian Chocó biogeographic, we constructed a data base of presence of species of lianas and vines reported to December 2012, from digital databases, records scientific literature and direct inspection of herbarium specimens considering ecosystem types (environments), from which, the composition and richness to any environment was determined. To evaluate the affinity in terms of family composition between types of environments, hierarchical clustering analysis, "Cluster" was developed using the Jaccard similarity coefficient. 41 families, 198 genera distributed in 700 species of lianas and vines for Chocó were recorded. The families better represented as for number of species and kinds were Bignoniaceae (75/34), Fabaceae (74/19), Sapindaceae (59/4) and Rubiaceae (40/14). There is a similarity of $72 \%$ in the composition of lianas and vines on the types of Colombian Chocó Biogeographic environment.
\end{abstract}

Key words: Presence-absence, similarity, Bignoniaceae, climbing, diversity.

\section{Introducción}

La distribución de los organismos es reflejo del efecto de factores históricos y ecológicos actuales como el clima, la topografía y las características químicas y físicas del suelo, entre otros (Ricklefs 1990); de allí que el análisis de los patrones de distribución de las especies es un tema central de la teoría ecológica y constituye una herramienta práctica para la conservación, permite conocer la diversidad biológica de determinado territorio, hipotetizar sobre cuáles son sus centros de diversificación, la evolución y dispersión de los taxa, así como sus características corológicas, de acuerdo con la heterogeneidad de sus hábitats (Challenger 1998).

Las lianas y bejucos son plantas trepadoras fijadas al suelo que son incapaces de mantenerse erguidas por sí mismas y necesitan soporte de los árboles para alcanzar el dosel, utilizando diferentes mecanismos como espinas, sarcillos, raíces adventicias (León 2009); inician su ciclo de vida en el suelo y jamás pierden conexión con este (Gentry 1985). Las lianas alcanzan un crecimiento secundario llegando a tener, excepcionalmente, más de $20 \mathrm{~cm}$ de diámetro y $200 \mathrm{~m}$ de longitud (Putz 1984; Gentry 1985).

\footnotetext{
${ }^{1}$ Universidad Tecnológica del Chocó, Grupo de Investigación Flora Chocoana, A.A. 292, Quibdó, Chocó, Colombia. yorleida16@homail.com, deivisabadia@ gmail.com

${ }^{2}$ Universidad Nacional de Colombia sede Medellín, Facultad de Ciencias Agropecuarias, Calle 59 A N 63-20, Medellín, Colombia. adjlema@unal.edu.co
} 
En los bosques tropicales, aproximadamente, entre el 10 y $45 \%$ de los individuos de plantas son lianas y/o bejucos, pudiendo alcanzar el segundo lugar en biomasa después de los árboles (Burhnam 2002, 2004; De Walt \& Chave 2004; Schnitzer 2005; Addo-Fordjour et al. 2008; Van der Heijden \& Phillips 2008), y representan cerca del 20-25\% de la diversidad florística total (Gentry 1982; Schnitzer \& Bongers 2002; Addo-Fordjour et al. 2008; Carrasco-Urra \& Gianoli 2009). Sin lianas el suelo de un bosque tropical perdería hasta un $40 \%$ de hojarasca (Hegarty 1990) y muchos animales sufrirían al escasear la comida y los refugios (Montgomery \& Sunquist 1978; Emmons \& Gentry 1983; Galetti \& Pedroni 1994 citado por Morellato \& Leitão-Filho 1996). Estructuralmente, las lianas constituyen una red natural de fibras tramadas entre los árboles que proveen estabilidad arquitectural al bosque (Putz 1984), aunque su presencia paradójicamente representa un fuerte perjuicio para la salud del árbol huésped (Putz 1984; Stevens 1987; Clark \& Clark 1990; Dillenburg et al. 1993).

La distribución y abundancia de las lianas varía con la ubicación geográfica de los bosques (Balfour \& Bond 1993; Burnham 2002, 2004; Parthasarathy et al. 2004), la precipitación promedio anual (Gentry 1991; Schnitzer 2005; Van der Heijden \& Phillips 2008) y la altura sobre el nivel del mar (Parthasarathy et al. 2004; Homeier et al. 2010), entre otros.

El Chocó biogeográfico es considerado un ecosistema único en el mundo (Mittermeier 1997, 1998), debido a su alta complejidad (Gentry 1988; He et al. 1996; Condit et al. 2000; Poveda et al. 2004; Rangel et al. 2004). Su historia geológica ha determinado patrones complejos de colonización. Al estar adyacente al Istmo de Panamá, se considera ruta de dispersión obligada y epicentro del intercambio de fauna y flora entre Norte y Suramérica en el Terciario (Hershkovitz 1972). Así mismo, corresponde a la zona de mayor representación del corredor de conservación Chocó-Manabí, la zona más importante en términos de biodiversidad del hot-spot o Ecorregión Terrestre Prioritaria (ETP) de Tumbes-Chocó-Magdalena (CEPF 2002), donde además se destacan su alto grado de endemismo de plantas (Gentry 1982) y su inusual diversidad biológica (Gentry 1978, 1982), presumiblemente relacionada con una marcada heterogeneidad ambiental y ecosistémica.

Según Rangel-Ch. (2004), aproximadamente $40 \%$ de los bosques naturales del Chocó
Biogeográfico, han sido deforestados principalmente por fragmentación del hábitat, explotación excesiva y sin planeación de especies maderables, la expansión de la frontera agrícola, la explotación de metales preciosos y la contaminación de las aguas y de los suelos por vertimiento de productos tóxicos o extraños al sistema natural.

A pesar de los diferentes estudios realizados sobre las lianas y del papel que cumplen en los bosques y su importancia para los animales, es poca la atención que se le presta a este grupo florístico. Linares (2001), afirma que el conocimiento sobre las lianas y bejucos en Colombia es bastante pobre debido a que la mayoría de los estudios florísticos se enfocan hacia la vegetación arbórea, además del hecho de que los métodos de investigación vegetales en campo no incluyen los relacionados con este grupo florístico.

En este trabajo se hizo una revisión de las bases de datos de diferentes herbarios colombianos y una revisión de artículos de revistas indexadas con el objetivo de determinar la composición a nivel de familia de lianas y bejucos en el Chocó biogeográfico.

\section{Materiales y Métodos}

Área de estudio: El Chocó Biogeográfico colombiano es una porción del territorio americano que representa solo un $2 \%$ de la superficie terrestre (175.000 Km²), sin embargo alberga cerca del 10\% de la biodiversidad del planeta (Diaz \& Gast 2009); corresponde a la zona de mayor representatividad del corredor de conservación Chocó-Manabí, la zona más importante en términos de biodiversidad de la Ecorregión Terrestre Prioritaria (ETP), del Tumbes-Chocó-Magdalena (CEPF 2002).

Varios intentos se han realizado para establecer un límite para el Chocó Biogeográfico colombiano, no obstante, a pesar de que sus contribuciones han diferido considerablemente, dependiendo principalmente del enfoque de estudio y del hecho que han considerado solo el fenómeno cultural (West 1957), el gradiente latitudinal (Hernández \& Sánchez 1992; Rodríguez-B. et al 1993; Rangel-Ch. et al 1995) o la división políticoadministrativa (IGAC 1999), terminan siendo complementarios entre si.

El Chocó biogeográfico colombiano está localizado entre el Océano Pacifico y la cordillera occidental, tomando la costa como eje de la limitación geográfica, entre los $8^{\circ} 30^{\prime}$ de latitud norte y $1^{\circ}$ de latitud norte (Rangel-Ch \& 
Arellano-P 2004), comprende geopolíticamente los departamentos del Chocó, Valle del Cauca, Cauca, Nariño y Antioquia (Fig. 1).

Abarca paisajes desde las tierras bajas con suelos aluviales a los lados de grandes ríos hasta colinas y serranías, con pendientes fuertes en la cabecera de los ríos y formadas por rocas ígneas con incidencia de basaltos diabasas y andesitas (Martínez 1993).

Existe una zonificación climática que considera tres subregiones: Norte $\left(8^{\circ} 30^{\prime}-6^{\circ} 30^{\prime}\right)$, centro $\left(<6^{\circ} 30^{\prime}\right.$ a $\left.3^{\circ} 30^{\prime}\right)$, sur $\left(>3^{\circ} 30^{\prime}\right.$ a $\left.1^{\circ}\right)$ con precipitación promedio anual de 2.526,9; 5.938,8 y 4.015,4 mm respectivamente. En cuanto a la humedad la zona centro presenta $87,71 \%$ de promedio anual de humedad relativa, la zona sur $87,65 \%$ y la zona norte $81,25 \%$. La temperatura media anual es mayor en el sector norte y corresponde a $27,42^{\circ} \mathrm{C}$ y disminuye hacia el centro y sur (Rangel-Ch \& Arellano-P 2004).

Colección: La especies de lianas y bejucos reportadas a diciembre de 2012, a partir de bases de datos digitales: Trópicos of Missouri Botanical Garden (MO), Field Museum of Natural History (F), New York Botanical Garden (NY), Smithsonian Institution (US), Herbario Nacional Colombiano (COL); literatura científica e inspección directa de especímenes del Herbario de la Universidad Tecnológica del Chocó (CHOCO) y del Herbario Universidad de Antioquia (HUA), se construyó una base de datos de registros de presencia ausencia de las especies de lianas y bejucos reportadas a diciembre del 2012, teniendo en cuenta los tipos de ecosistemas (ambientes), descritos por RangelCh. (2004), (Estuarino, Fluvio Lacustre, Llanura aluvial, Terrazas bajas, Colinas bajas, Colinas medias, Colinas altas, Subandino).

Cada registro de la base de datos fue georeferenciado de acuerdo con la información disponible en los especímenes. En caso de que esta información no estuviera en los especímenes se utilizaron gaceteros digitales, Geonames, Global Gazetteer Version 2.3, Google Earth y datos bibliográficos.

Riqueza y composición familias de lianas y bejucos entre los tipos de ecosistema (Ambientes)

A pesar de que existen varias propuestas de subdivisión del Chocó biogeográfico, para este estudio, se acogió la clasificación de tipos de ecosistemas propuestos por Rangel-Ch. (2004), por presentar un enfoque integrador con base en componentes geográficos, que incluyen la fisiografía, influencia de los factores hídricos, topográficos, y bióticos como asociaciones vegetales que reflejan las influencia de las condiciones medioambientales, como sigue: Estuarios $(0-10 \mathrm{~m})$. Zona con influencia del agua del mar y de la desembocadura de los ríos. Fluvio lacustre $(0-10 \mathrm{~m})$. Zonas influenciadas por los caudales del río, quebradas u otro tipo de corrientes. Llanura aluvial (entre 10-50 m). Comprende las áreas sometidas a la inundación por acción de los ríos. Terrazas bajas (>50-100 m); Colinas bajas (>100-250 m); Colinas medias $(>250-500$ $\mathrm{m}$ ); Colinas altas (serranías $>500-1.000 \mathrm{~m}$ ) y Subandino cordillerano (Premontano o Subandino $>1.000-2.300 \mathrm{~m}$ ).

Para evaluar la afinidad en términos de composición de familias entre tipos de ambientes, se elaboró un análisis de agrupamiento jerárquico, utilizando el coeficiente de similaridad de Jaccard. Para este análisis solo se tuvieron en cuenta aquellas familias presentes en cuatro o más tipos de ambiente, para evitar el efecto de datos raros (familias con solo uno, dos o tres registros); Este análisis se realizó con el paquete estadístico PAST (Versión 2.17). Se corrió una regresión de Poisson en Statgrafics para determinar si existen diferencias estadísticamente significativas entre el número de familias con respecto a los ambientes como variables independientes; este modelo permite estimar el número de especies por ambientes que se encontraran multiplicadas. Adicional a lo anterior se corrieron modelos lineales generalizados para ver si conjuntamente número de especies, géneros y familias dependían de los ambientes. Para detectar si existe autocorrelación entre los residuales del modelo se calculó en estadístico Durbin-Watson, se estimaron los intervalos de confianza para las medias de número de especies en cada familia.

\section{Resultados}

Riqueza y composición de familias de lianas y bejucos

Se registró un total de 2824 individuos de lianas y bejucos distribuidos en, 41 familias, 198 géneros y 700 especies para el Chocó Biogeográfico (Material Suplementario).

Las familias mejor representadas fueron Bignoniaceae (con 75 especies), Fabaceae (74), Sapindaceae (59), Rubiaceae (40), Passifloraceae 
(40). El patrón de riqueza demuestra que en las 15 familias mejor representadas de lianas y bejucos se concentra el 79\% de las especies y el 78\% de los géneros de este grupo florístico. (Tab.1)

Se registraron 198 géneros entre los cuales sobresalen por ser los más abundantes Passiflora (con 157 especies), Paullinia (124), Anemopaegma
(120), Schlegelia (100). La Figura 2 muestra las especies más abundantes.

A nivel de especies, Tanaecium pyramidatum, Passiflora vitifolia, Sabicea panamensis, Sabicea villosa y Schlegelia parviflora estuvieron presente en todos los ambientes descritos para el Chocó Biogeográfico Colombiano (Fig. 3).

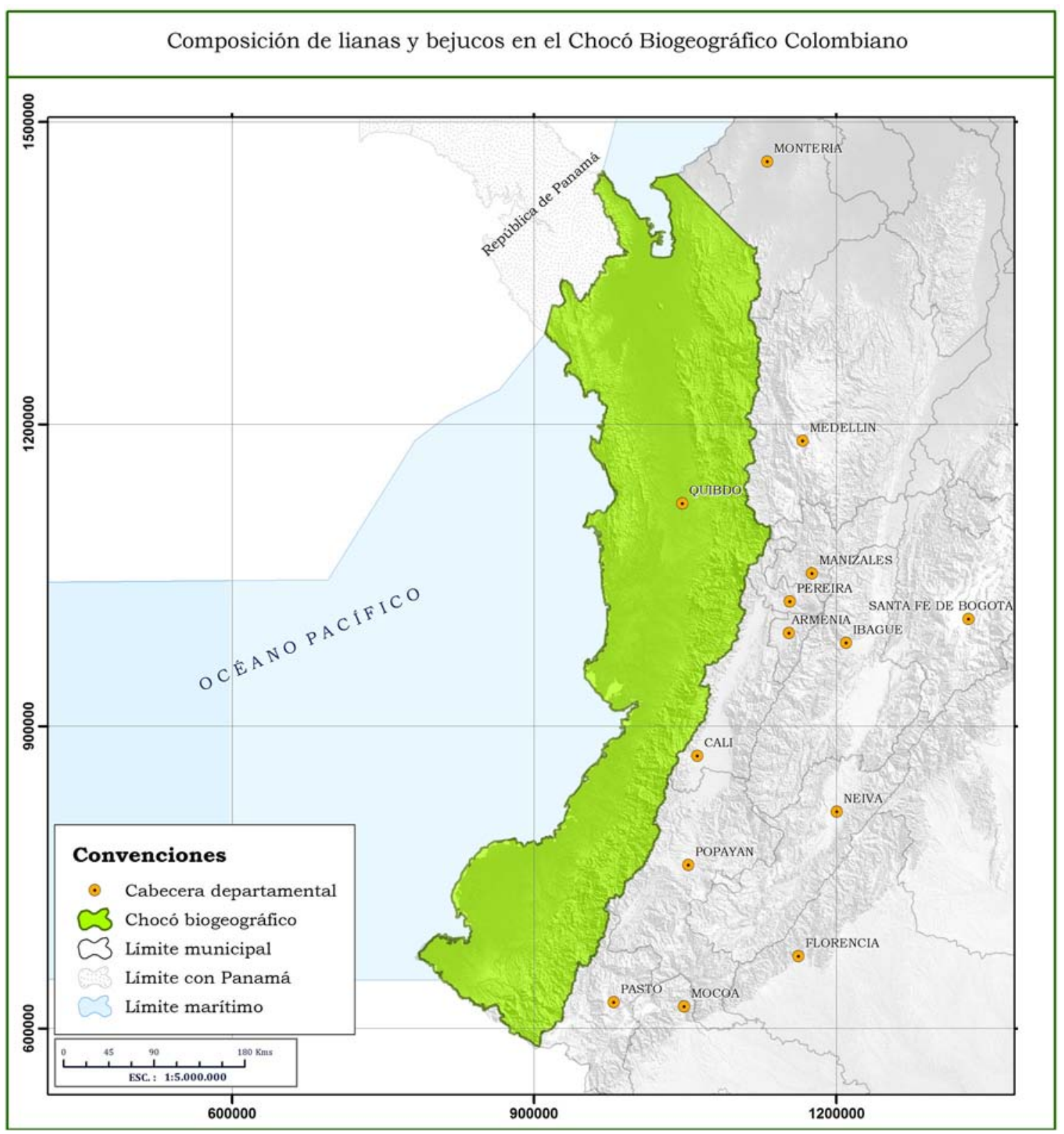

Figura 1 - Ubicación geográfica del Chocó Biogeográfico Colombiano.

Figure 1 - Geographical location of Chocó Biogeographic Colombiano. 
Distribución de la Riqueza de familias de lianas y bejucos entre los tipos de ecosistema (Ambientes)

El análisis de la distribución de lianas y bejucos en el Chocó biogeográfico colombiano muestra los valores más altos de riqueza de lianas y bejucos se presentan a nivel de familias en los ambientes de Colinas medias (38), Colinas bajas (37), Llanura aluvial (37) y Terrazas bajas (34). Sin embargo, a nivel de especies y géneros se destacan Llanura aluvial (294/134), Colinas bajas (291/143), Terrazas bajas (229/117) (Fig. 4).

Quince familias están presentes en los ocho tipos de ecosistemas del Chocó biogeográfico, destacándose Bignoniaceae, Fabaceae y Sapindaceae con 75/34, 74/19 y 59/4 especies y géneros respectivamente. Amaranthaceae fue exclusiva del ambiente de colinas medias.

Geopolíticamente la región del Chocó biogeográfico colombiano está integrada por territorios de los departamentos del Chocó, Valle del Cauca, Cauca, Nariño y Antioquia, Rangel (2004). Se, registraron 41 familias, las cuales se encuentran distribuidas en Antioquia, Cauca, Chocó, Nariño, Risaralda y Valle del Cauca. El departamento donde estuvieron presentes todas las familias fue el departamento del Chocó, mientras que Risaralda solo registro dos familias. Antioquia se registraron 23, Cauca 25, Nariño 26 y Valle del Cauca 29. Las familias; Amaranthaceae, Combretaceae, Gnetaceae, Rhamnaceae, Trigoniaceae, Ulmaceae, Violaceae, Icacinaceae fueron exclusivas del departamento del Chocó.

Tabla 1 - Distribución de la riqueza de familias de lianas y bejucos por ambientes en el Chocó biogeográfico Colombiano. Sp: especie, G: genero

Table 1 - Distribution of wealth family's lianas and vines for environments in Chocó biogeographic Colombiano. Sp: species, G: gender

\begin{tabular}{|c|c|c|c|c|c|c|c|c|}
\hline & $\begin{array}{c}\text { Colinas } \\
\text { Altas }\end{array}$ & $\begin{array}{c}\text { Colinas } \\
\text { Bajas }\end{array}$ & $\begin{array}{l}\text { Colin a s } \\
\text { Medias }\end{array}$ & Estuarino & $\begin{array}{c}\text { F l u v i o } \\
\text { Lacustre }\end{array}$ & $\begin{array}{c}\text { L I a n u r a } \\
\text { Aluvial }\end{array}$ & Subandino & $\begin{array}{c}\text { Terrazas } \\
\text { Bajas }\end{array}$ \\
\hline Familia & Sp-G & Sp-G & Sp-G & Sp-G & Sp-G & Sp-G & Sp-G & Sp-G \\
\hline Acanthaceae & & $1-1$ & $1-1$ & & $2-1$ & $2-1$ & & \\
\hline Amaranthaceae & & & $1-1$ & & & & & \\
\hline Apocynaceae & $9-7$ & $16-12$ & $9-6$ & $9-8$ & $8-7$ & $18-10$ & $3-1$ & $15-9$ \\
\hline Arecaceae & & $1-1$ & & & & $1-1$ & & \\
\hline Aristolochiaceae & $3-1$ & $8-1$ & $4-1$ & $5-1$ & $3-1$ & $6-1$ & $6-1$ & $7-1$ \\
\hline Asclepiadaceae & $3-3$ & $7-6$ & $2-2$ & $3-2$ & $1-1$ & $6-4$ & $10-6$ & $3-3$ \\
\hline Asteraceae & $15-8$ & $5-4$ & $5-2$ & $4-1$ & $3-2$ & $9-4$ & $6-3$ & $8-5$ \\
\hline Bignoniaceae & $30-20$ & $44-26$ & $27-19$ & $17-13$ & $23-16$ & $43-22$ & $27-17$ & $23-16$ \\
\hline Boraginaceae & $5-1$ & $2-1$ & $2-1$ & $2-1$ & $1-1$ & $4-1$ & $1-1$ & $3-1$ \\
\hline Campanulaceae & $1-1$ & & $2-1$ & & & $1-1$ & $1-1$ & \\
\hline Combretaceae & & $2-2$ & & & $1-1$ & $1-1$ & & $1-1$ \\
\hline Commelinaceae & $2-1$ & $4-1$ & $2-1$ & $1-1$ & & $3-1$ & $1-1$ & $1-1$ \\
\hline Connaraceae & & $2-2$ & $2-2$ & $1-1$ & $3-2$ & $4-4$ & & $3-1$ \\
\hline Convolvulaceae & $6-5$ & $14-7$ & $4-2$ & $9-2$ & $7-3$ & $6-2$ & & $9-5$ \\
\hline Cucurbitaceae & $14-9$ & $18-11$ & $14-8$ & $3-3$ & $5-4$ & $15-10$ & $2-1$ & $16-9$ \\
\hline Сyperaceae & $2-1$ & $4-1$ & $2-1$ & $2-1$ & $3-1$ & $6-1$ & & $3-1$ \\
\hline Dichapetalaceae & & $2-1$ & $2-1$ & & $1-1$ & $4-1$ & & $1-1$ \\
\hline Dilleniaceae & $2-2$ & $7-4$ & $3-3$ & $4-3$ & $4-2$ & $6-3$ & & $8-3$ \\
\hline Dioscoriaceae & & $2-1$ & $3-1$ & $2-1$ & & $2-1$ & $2-1$ & $1-1$ \\
\hline Euphorbiaceae & $2-1$ & $7-3$ & $3-3$ & $2-2$ & $1-1$ & $5-4$ & & $4-3$ \\
\hline
\end{tabular}




\begin{tabular}{lcccccccc}
\hline & $\begin{array}{c}\text { Colinas } \\
\text { Altas }\end{array}$ & $\begin{array}{c}\text { Colinas a } \\
\text { Bajas }\end{array}$ & $\begin{array}{c}\text { C o l in a s } \\
\text { Medias }\end{array}$ & Estuarino & $\begin{array}{c}\text { F l u v i o } \\
\text { Lacustre }\end{array}$ & $\begin{array}{c}\text { L l a n u r a } \\
\text { Aluvial }\end{array}$ & $\begin{array}{c}\text { Subandino } \\
\text { Berrazas } \\
\text { Bajas }\end{array}$ \\
\hline Fabaceae & $25-9$ & $20-12$ & $15-9$ & $13-6$ & $17-10$ & $27-15$ & $1-2$ & $29-12$ \\
Gnetaceae & $1-1$ & & $1-1$ & & & $1-1$ & & \\
Hippocrateaceae & $2-2$ & $9-6$ & $2-2$ & $2-2$ & $1-1$ & $1-1$ & & $4-2$ \\
Icacinaceae & & & $1-1$ & & & $1-1$ & & \\
Loganiaceae & & $7-1$ & $5-1$ & $6-1$ & $3-1$ & $8-1$ & $1-1$ & $4-1$ \\
Malpighiaceae & $3-2$ & $6-5$ & $9-6$ & $8-4$ & $7-4$ & $20-6$ & $3-3$ & $11-5$ \\
Melastomataceae & $3-2$ & $1-1$ & $3-2$ & & $3-2$ & $4-2$ & $1-1$ & $2-2$ \\
Menispermaceae & $3-3$ & $10-7$ & $5-4$ & $4-3$ & $2-3$ & $11-7$ & & $3-3$ \\
Olacaceae & $2-1$ & $4-1$ & $2-1$ & $1-1$ & $1-1$ & $3-1$ & & $3-1$ \\
Passifloraceae & $11-1$ & $20-2$ & $13-1$ & $6-1$ & $13-1$ & $16-2$ & $6-1$ & $17-2$ \\
Polygalaceae & & $1-1$ & $1-1$ & $1-1$ & & & & \\
Polygonaceae & $3-1$ & $3-1$ & $5-1$ & $3-1$ & $1-1$ & $2-1$ & & $3-2$ \\
Rhamnaceae & $1-1$ & $1-1$ & $2-1$ & & $2-1$ & $2-1$ & & $1-1$ \\
Rubiaceae & $7-5$ & $18-8$ & $8-7$ & $6-5$ & $5-3$ & $18-7$ & $6-4$ & $15-9$ \\
Sapindaceae & $16-3$ & $19-3$ & $10-3$ & $9-1$ & $11-2$ & $19-3$ & $20-4$ & $8-2$ \\
Smilacaceae & $4-1$ & $3-1$ & $2-1$ & $1-1$ & $1-1$ & $6-1$ & $7-1$ & $2-1$ \\
Trigoniaceae & & $1-1$ & & & & $1-1$ & & $1-1$ \\
Ulmaceae & & $1-1$ & $1-1$ & & & & & $1-1$ \\
Verbenaceae & $8-1$ & $7-2$ & $5-2$ & $2-1$ & $2-2$ & $5-1$ & $2-1$ & $4-1$ \\
Violaceae & & $1-1$ & $1-1$ & $1-1$ & & & & $1-1$ \\
Vitaceae & $5-2$ & $6-2$ & $2-1$ & $4-1$ & $2-1$ & $8-2$ & $1-1$ & $6-2$ \\
Total general & $188-95$ & $284-142$ & $181-103$ & $131-70$ & $137-78$ & $295-127$ & $107-52$ & $221-110$ \\
\hline & & & & & & & &
\end{tabular}

El departamento que más individuos de lianas y bejucos registro fue el departamento del Chocó, con 1.897 individuos, seguido del Valle del Cauca con 449, Antioquia 194, Nariño 159, Cauca 121; el departamento con menos individuos fue Risaralda, con tres individuos registrados a través de esta investigación.

En cuanto a géneros, el departamento del Chocó registró (189 géneros), Valle (90), Nariño (57), Antioquia (56), Cauca (47) y Risaralda (3). 20 géneros son exclusivos para el departamento del Chocó.El agrupamiento jerárquico, mostró una alta similaridad entre los tipos de ambientes (72\%) en los arreglos espaciales de familias de lianas y bejucos, lo que permite reconocer con claridad un grupo conformado por los ambientes de colinas bajas y terrazas bajas con un poco más de $90 \%$ de similaridad, que a su vez, se relaciona en un 80\% con el ambiente de llanura aluvial (Fig. 5).
El estadístico R-Cuadrada indica que el modelo, explica $78.7138 \%$ de la variabilidad en nues, mientras que para número de géneros, R-Cuadrada ajustada explica el $85.2285 \%$. El estadístico de Durbin-Watson (DW) prueba los residuos para determinar si hay alguna correlación significativa con base en el orden en el que se presentaron en el archivo de datos. Puesto que el valor-P es menor que 0,05 hay indicación de una posible correlación serial.

El análisis de ANOVA multivariado (MANOVA) se usa para determinar si un efecto en particular tiene una relación significativa con el grupo de variables dependientes que está siendo modelado. Valores-P menor de 0,05 indican que hay un efecto estadísticamente significativo al nivel de confianza del 95,0\%. En este caso, el factor más importante (de acuerdo con la lambda de Wilks) es familia. 


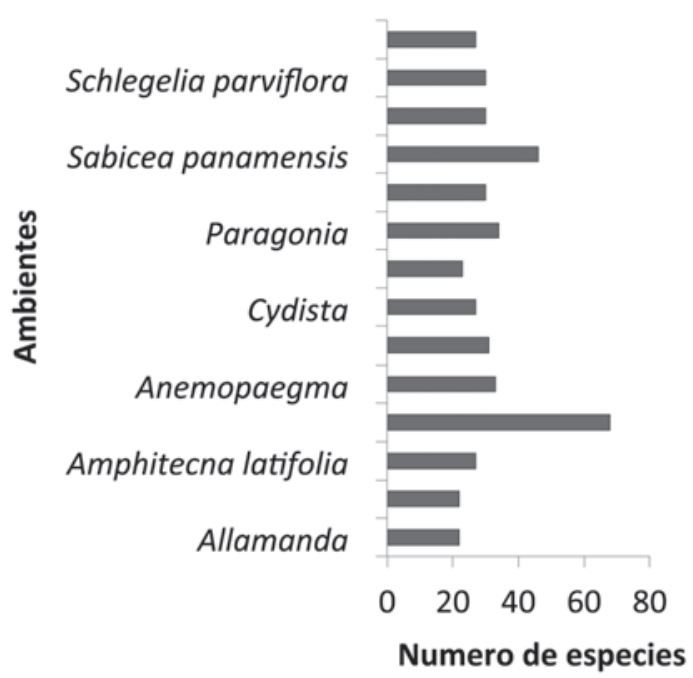

Figura 2 - Especies de lianas y bejucos más abundantes en el Chocó Biogeográfico Colombiano

Figure 2 - Species of lianas and vines more abundant in the Colombian Chocó

\section{Discusión}

Linares (2001), reportó para Colombia 70 familias de bejucos; mientras que Rangel-Ch. \& Rivera (2004) registraron un total de 170 familias de espermatofitos para el Chocó biogeográfico. Teniendo en cuenta nuestros resultados; el 21\% de las familias de espermatofitos del Chocó Biogeográfico son lianas y bejucos o incluyen generos y/o especies de este grupo. Esta alta representatividad ha sido documentada por muchos autores en los bosques tropicales (Gentry 1982; Schnitzer \& Bongers 2002; Burhnam 2002, 2004; De Walt \& Chave 2004; Schnitzer 2005; Addo-Fordjour et al. 2008; Van der Heijden \& Phillips 2008; Carrasco-Urra \& Gianoli 2009; entre otros).

Schnitzer et al (2012), en un estudio realizado en la isla de Barro Colorado en Panamá sobre abundancia, diversidad y distribución de lianas, encontaron que las familias más diversas fueron Sapindaceae, Fabaceae, Apocynaceae, Bignoniaceae y Malpighiaceae; En otro estudio de lianas en la estación ecológica Noroeste de Paulista, Brasil, Rezende \& Ranga (2005) reportaron las mismas familias como las más diversas.

La distribución a nivel de familias de lianas y bejucos en el Chocó biogeográfico posiblemente está relacionada con el hecho de que estos ambientes tipifican de alguna manera la zona denominada depresión pacifica o anden pacifico (Rangel-Ch. \& Rivera 2004). No obstante, esta aseveración debe ser tomada con cierto grado de reserva, debido a la poca disponibilidad de información sobre este grupo de plantas en el Chocó biogeográfico, pues es mucho más probable encontrar registros de especies arbóreas, ya que en su gran mayoría los estudios florísticos han estado focalizados hacia este hábito (especialmente $>10 \mathrm{~cm}$ ) y a la poca atención que se ha dado a las lianas y bejucos, a pesar de su gran importancia en la dinámica y el gran aporte que se ha manifestado realizan a la diversidad florística en los bosques tropicales.

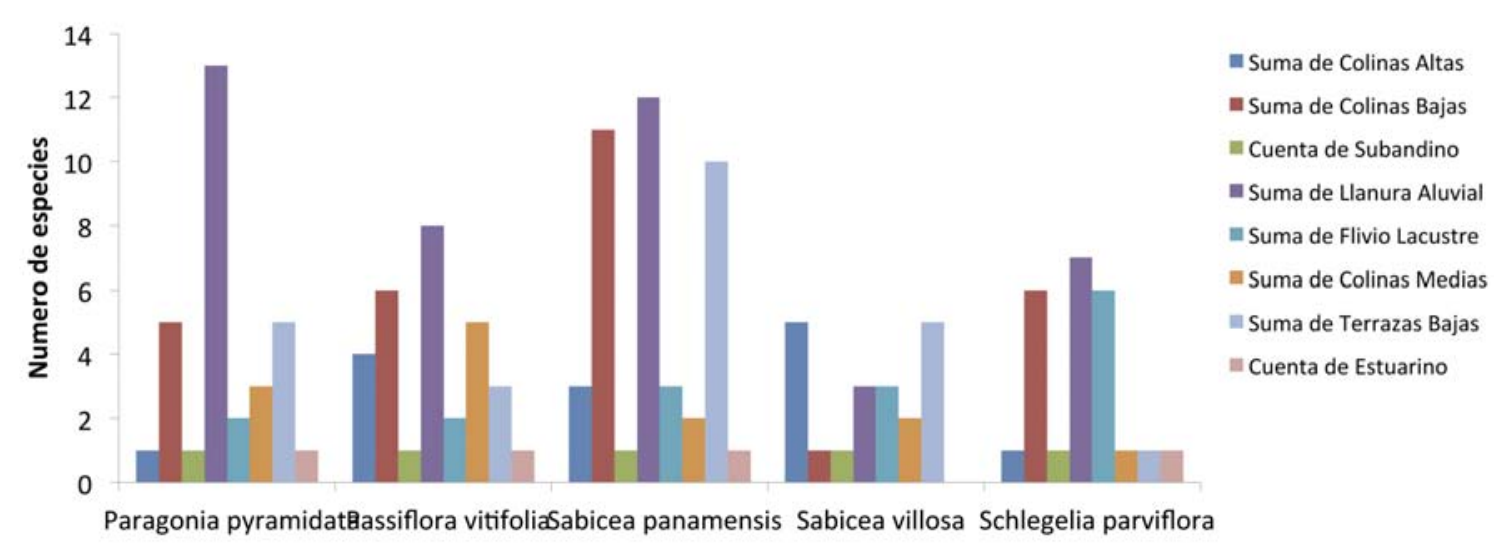

Figura 3 - Especies de lianas y bejucos presentes en todos los ambientes del Chocó Biogeográfico colombiano Figure 3 - Species of lianas and vines present in all environments Colombian Chocó Biogeographic 


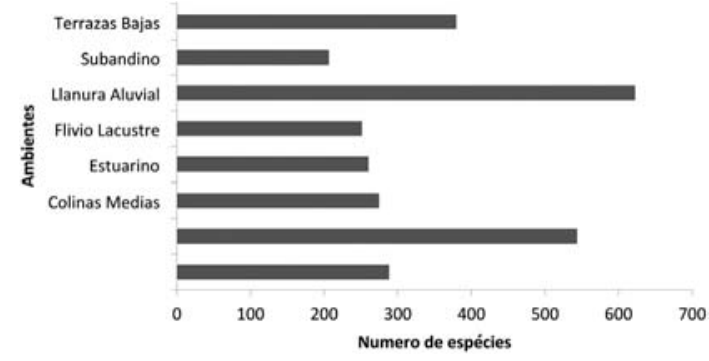

Figura 4 - Abundancia de especies de lianas y bejucos por ambiente en el Chocó Biogeográfico Colombiano. Figure 4 - Abundance of species of lianas and vines by environment in Colombian Chocó.

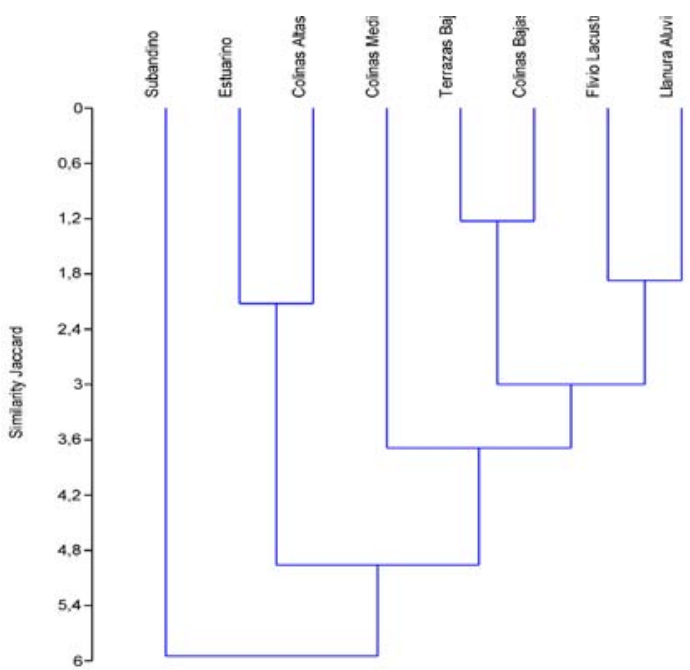

Figura 5 - Similaridad entre los tipos de ambientes a nivel de familias en el Chocó Biogeográfico Colombiano. Figure 5 - Similarity between level environments types of families in the Colombian Chocó.

Por lo tanto, estos resultados deben ser interpretados como preliminares, pues, para poder realizar aseveraciones contundentes se requiere de más información tanto a nivel de registros de especímenes como de las variables ambientales y su influencia sobre la distribución de la riqueza y diversidad de especies.

\section{Conclusiones}

Con 41 familias, 198 géneros y 700 especies de lianas y bejucos, el Chocó Biogeográfico Colombiano se podría identificar como una región potencialmente diversa para este grupo de plantas, ya que con tan solo el $7,50 \%$ del territorio colombiano, alberga el 58,5\% de la diversidad de familias para este grupo en el país.

Para el Chocó Biogeográfico Colombiano, las familias Bignoniaceae, Fabaceae, y Sapindaceae con 75, 74 y 59 especies respectivamente, agrupan 208 de las 700 especies registradas, son las que más aportan a la diversidad de lianas y bejucos de la región.

Igualmente se destaca el hecho de que los ambientes que concentran la mayor cantidad de familias son Colinas medias (38), Llanura aluvial (37) y Colinas bajas (37); mientras que para géneros y especies Llanura aluvial (134/294), Colinas bajas (143/291) y Terrazas bajas (117/229) son los más importantes. Las lianas y bejucos presentan una alta similaridad entre los tipos de ambientes $72 \%$ a nivel de familias. Sin embargo, las familias Acanthaceae, Apocynaceae, Bignoniaceae, Boraginaceae, Campanulaceae, Combretaceae, Convolvulaceae, Cucurbitaceae, Fabaceae, Hippocrateaceae, Passifloraceae, Polygonaceae, Rhamnaceae, Sapindaceae, marcan una diferencia importante en cuanto a su distribución en los tipos de ambiente del Chocó Biogeográfico colombiano; mientras que, los tipos de ambiente, Colinas bajas, Subandino y Colinas altas, son muy diferentes.

\section{Agradecimientos}

A la Universidad Tecnológica del Chocó “Diego Luis Córdoba” por la financiación del proyecto, al Centro de Investigaciones en Biodiversidad y Hábitat de la Universidad Tecnológica del Chocó y al Grupo de Investigación de la Flora Chocoana.

\section{Referencias}

Bongers, F.J.J.M; Schnitzer, S.A. \& Traoré, D. 2002. The importance of lianas and consequences for forest management in West Africa Bioterre: revue internationale scientifique de la vie et de la terre Pp. 59-70.

Burnham, R.J. 2002. Dominance, diversity and distribution of lianas in Yasuni, Ecuador: who is on top? Journal of Tropical Ecology 18:845-864.

Burnham, R.J. 2004. Alpha and beta diversity of lianas in Yasuní, Ecuador. Forest Ecology and Management 190: 43-55.

Challenger, A. 1998. Utilización y conservación de vegetación de los ecosistemas terrestres de México. Pasado, presente y futuro. Instituto de Biología, Universidad Nacional Autónoma de México y 
Agrupación Sierra Madre S.C. Comisión Nacional para el conocimiento y uso de la Biodiversidad, México. 847p.

Clark, D.B. \& Clark D.A. 1990. Distribution and effects on tree growth of lianas and woody hemiepiphytes in a Costa Rican Tropical Wet Forest. Journal of Tropical Ecology 6: 321-331.

Dillenburg, L.R.; Whigham, D.F.; Teramura, A.H. \& Forseth, I.N. 1993. Effects of below- and aboveground competition from the vines Lonicera japonica and Parthenocissus quinquefolia on the growth of the tree host Liquidambar styraciflua. Oecologia 93: 48-54.

Dewalt, S.J.; Chave, J. 2004. Structure and biomass of four lowland Neotropical forests. Biotropica 36:7-19.

Emmons, L.H. \& Gentry, A.H. 1983. Tropical forest structure and the distribution of gliding and prehensile-tailed vertebrates. American Naturalist 121: 513-523.

Gentry, A.H. 1991. The distribution and evolution of climbing plants. In: Putz, F.E. \& Mooney, H.A. (eds.). The Bioogy of Vines. Cambridge University Press, Cambridge. Pp. 3-52

Gentry, A.H. 1982. Patterns of Neotropical plant species diversity. Evolutionary Biology 15: 1-84.

Hernández, J. \& Sánchez, H. 1992. Biomas terrestres de Colombia. In: Halffter G. (ed.). La diversidad biológica de Iberoamérica I. Vol. I. Instituto Nacional de ecología, secretaria de desarrollo social, México. 363p.

Hegarty, E.E. 1991. Vine-host interactions. In: Putz. F.E. \& Mooney, H.A. (eds.). The Biology of Vines. Cambridge University Press, Cambridge. Pp. 357-376.

Gentry, A.H. \& Dodson, C. 1987. Contribuition of nontrees to species richness of a tropical rain forest. Biotropica 19: 149-156.

Instituto Geográfico Agustín Codazzi y Ministerio del Medio Ambiente. 1999. Proyecto zonificación ecológica de la Región Pacifica colombiana. Biogeografía, Santafé de Bogotá. 96p.

Janzen, D.H. 1975. Ecology of plants in the Tropics. Studies in biology $n^{\circ}$. 58. The Institute of Biology. Edward Arnold Ltd., London. Pp. 1-15.

Linares, E. 2001. Aproximación al conocimiento de los bejucos en Colombia. Caldasia 23: 169-179.

Martínez, J.O. 1993. Geomorfología. In: Leyva P. (ed.). Colombia Pacifico Tomo I. Fondo para la protección del medio ambiente José Celestino Mutis, Bogotá. Pp. 110-119.

Morellato, L.P. \& Leitão, H.F. 1996. Fenología reproductiva de los escaladores en un bosque brasileño sudeste. Biotropica 28: 180-191.

Putz, F. 1984. The natural history of lianas on Barro Colorado Island. Ecology 65: 1713-1724.

Poveda, I.C.; Rojas, C.A.; Rudas, A. \& Rangel-Ch, J.O. 2004. El Chocó Biogeográfico: ambiente físico. In: Rangel-Ch, J.O. (ed.). Colombia Diversidad Biótica IV: El Chocó Biogeográfico/Costa pacífica. Instituto de Ciencias Naturales. Universidad Nacional de Colombia, Bogotá. Pp. 1-22.

Rangel-Ch, J.O. \& Rivera, O. 2004a. Diversidad y riqueza de espermatofitos $\mathrm{In}$ : Rangel-Ch, J.O. (ed.). Colombia diversidad biótica IV: el Chocó Biogeográfico/Costa pacífica. Instituto de Ciencias Naturales. Universidad Nacional de Colombia, Bogotá. Pp. 83-104

Rangel-Ch, J.O. 2004b. La vegetación del Chocó Biogeográfico de Colombia y zonas cordilleranas aledañas - síntesis. In: Rangel-Ch, J.O. (ed.). Colombia diversidad biótica IV: El Chocó Biogeográfico/Costa pacífica. Instituto de Ciencias Naturales. Universidad Nacional de Colombia. Bogotá. Pp. 769-815.

Rezende, A.A. \& Ranga, N.T. 2005. Lianas da Estação Ecológica do Noroeste Paulista, São José do Rio Preto/Mirassol, SP, Brasil. Acta Botanica Brasilica 19: 273-279.

Rangel-Ch., J.O. \& Arellano, H. 2004. Clima del Chocó biogeográfico. In: Rangel-Ch., J.O. (ed.). Colombia Diversidad Biótica IV. El Chocó biogeográfico/ Costa Pacífica. Instituto de Ciencias Naturales, Bogotá D.C. Pp. 39-82.

Schnitzer, A.; Mangan, S.A.; Dalling, J.W.; Baldeck, C.A.; Hubbell, S.P.; Ledo, A.; Muller-Landau, H.; Tobin, M.F. ; Aguilar, S.; Brassfield, D.; Hernandez, A.; Lao, S.; Perez, R.; Valdes, O. \& Yorke, S.R. 2012. Liana abundance, diversity, and distribution on Barro Colorado Island, Panama. Plos one 7: 1-16.

Toledo, A.T. 2010. Las lianas y la dinámica de los bosques tropicales. Universidad Autónoma de México. Ciencias 98: 15-20.

Van der Heijden, G.M.F. \& Phillips, O.L. 2008. What controls liana success in Neotropical forests? Global Ecology and Biogeography 17: 372-383. 
